\title{
Correction to: effects of dietary supplementation of a lipid-coated zinc oxide product on the fecal consistency, growth, and morphology of the intestinal mucosa of weanling pigs
}

Young-Jin Byun ${ }^{1 \dagger}$, Chul Young Lee ${ }^{1 \dagger}$, Myeong Hyeon Kim¹, Dae Yun Jung ${ }^{1}$, Jeong Hee Han², Insurk Jang ${ }^{3}$, Young Min Song ${ }^{1}$ and Byung-Chul Park ${ }^{4 *}$

\section{Correction}

Due to a technical issue this article [1] was accidentally published in volume 59, the correct volume for this article is volume 60 .

\begin{abstract}
Author details
'Department of Animal Resources Technology, Gyeongnam National University of Science and Technology, Jinju 52725, South Korea. ${ }^{2}$ College of Veterinary Medicine and Institute of Veterinary Science, Kangwon National University, Chuncheon 24341, South Korea. ${ }^{3}$ Department of Animal Science and Biotechnology, Gyeongnam National University of Science and Technology, Jinju 52725, South Korea. ${ }^{4}$ Graduate School of International Agricultural Technology, Institute of Green Bio Science and Technology, Seoul National University, Pyeongchang 25354, South Korea.
\end{abstract}

Received: 9 February 2018 Accepted: 12 February 2018

Published online: 05 March 2018

\section{Reference}

1. Byun YJ, et al. Effects of dietary supplementation of a lipid-coated zinc oxide product on the fecal consistency, growth, and morphology of the intestinal mucosa of weanling pigs. J Anim Sci Technol. 2017;60:29. https:// doi.org/10.1186/s40781-017-0159-z.

\footnotetext{
* Correspondence: bcpark@snu.ac.kr

${ }^{\dagger}$ Equal contributors

${ }^{4}$ Graduate School of International Agricultural Technology, Institute of Green Bio Science and Technology, Seoul National University, Pyeongchang 25354, South Korea
} 